\title{
Simulating the detailed chemical composition of secondary organic aerosol formed on a regional scale during the TORCH 2003 campaign in the southern UK
}

\author{
D. Johnson ${ }^{1}$, S. R. Utembe ${ }^{1}$, and M. E. Jenkin ${ }^{1}$ \\ ${ }^{1}$ Centre for Environmental Policy, Imperial College London, Silwood Park, Ascot, Berkshire, SL5 7PY, UK
}

Received: 18 July 2005 - Published in Atmos. Chem. Phys. Discuss.: 30 August 2005

Revised: 5 December 2005 - Accepted: 10 January 2005 - Published: 8 February 2006

\begin{abstract}
Following on from the companion study (Johnson et al., 2006), a photochemical trajectory model (PTM) has been used to simulate the chemical composition of organic aerosol for selected events during the 2003 TORCH (Tropospheric Organic Chemistry Experiment) field campaign. The PTM incorporates the speciated emissions of 124 nonmethane anthropogenic volatile organic compounds (VOC) and three representative biogenic VOC, a highly-detailed representation of the atmospheric degradation of these VOC, the emission of primary organic aerosol (POA) material and the formation of secondary organic aerosol (SOA) material. SOA formation was represented by the transfer of semiand non-volatile oxidation products from the gas-phase to a condensed organic aerosol-phase, according to estimated thermodynamic equilibrium phase-partitioning characteristics for around 2000 reaction products. After significantly scaling all phase-partitioning coefficients, and assuming a persistent background organic aerosol (both required in order to match the observed organic aerosol loadings), the detailed chemical composition of the simulated SOA has been investigated in terms of intermediate oxygenated species in the Master Chemical Mechanism, version 3.1 (MCM v3.1). For the various case studies considered, $90 \%$ of the simulated SOA mass comprises between ca. 70 and 100 multifunctional oxygenated species derived, in varying amounts, from the photooxidation of VOC of anthropogenic and biogenic origin. The anthropogenic contribution is dominated by aromatic hydrocarbons and the biogenic contribution by $\alpha$ - and $\beta$-pinene (which also constitute surrogates for other emitted monoterpene species). Sensitivity in the simulated mass of SOA to changes in the emission rates of anthropogenic and
\end{abstract}

Correspondence to: M. E. Jenkin

(m.jenkin@imperial.ac.uk) biogenic VOC has also been investigated for 11 case study events, and the results have been compared to the detailed chemical composition data. The role of accretion chemistry in SOA formation, and its implications for the results of the present investigation, is discussed.

\section{Introduction}

Atmospheric aerosol particulate material comprises variable mixtures of inorganic salts, crustal minerals, elemental carbon, organic carbon and water (e.g. Seinfeld and Pankow, 2003; Kanakidou et al., 2005). Where the mineral and inorganic component of atmospheric aerosol is relatively simple in composition, and hence may be effectively chemically characterised, the organic content is many orders of magnitude more complex. Primary organic aerosol (POA) material is emitted, along with elemental carbon (EC), directly into Earth's atmosphere from a large variety of combustion sources, and also from a number of non-combustion sources (e.g., plant debris suspension; wave and bubble breaking over water; virus and spore suspension; suspension of soil particles). Secondary organic aerosol (SOA) material is produced in situ from the atmospheric oxidation of emitted volatile organic compounds (VOC) following the transfer of semiand non-volatile reaction products from the gas-phase to a condensed organic particle-phase. Atmospheric SOA is thus composed of a myriad of different products of the oxidation of a variety of VOC, which are emitted to the Earth's atmosphere as a result of anthropogenic activity (e.g. aromatic hydrocarbons) and from globally distributed biogenic sources (e.g. monoterpene species) (see, e.g., Seinfeld and Pandis, 1998; Finlayson-Pitts and Pitts, 1999). It is not

(C) 2006 Author(s). This work is licensed under a Creative Commons License. 
currently possible to fully chemically characterise the organic aerosol (OA) component of atmospheric particulate material and yet OA comprises a very significant mass fraction of fine-particles (diameters $\leq 2.0 \mu \mathrm{m}$ ) which are responsible for nearly all aerosol-effects of atmospheric concern. Aerosol particulate material can affect the Earth's climate directly by absorbing or scattering solar radiation, or indirectly by altering the properties and lifetimes of clouds (IPCC, 2001). Fine particulate material is also implicated in adverse health effects in humans - fine particles are able to penetrate deep into the respiratory tract and lungs - as a result of epidemiologic studies (e.g. Schwartz et al., 1996; Laden et al., 2000; Anderson et al., 2001; Englert, 2004). In order to begin to understand the mechanisms by which these important atmospheric effects are brought about, it is first necessary to better understand the detailed chemical content of atmospheric particulate material in terms of the chemical structures, solubilities, other physical properties and reactivities of the various classes of constituent species (e.g. inorganic salt, organic component, etc.) and of the individual components themselves.

In late July and August 2003 the first field measurement campaign of the collaborative research project TORCH (Tropospheric Organic Chemistry Experiment) was conducted. As described in greater detail by Utembe at al. (2005) and in the companion to this paper (Johnson et al., 2006), a variety of measurements were made at Writtle College in the Southern UK $\left(51^{\circ} 44^{\prime} 12^{\prime \prime} \mathrm{N} ; 0^{\circ} 25^{\prime} 28^{\prime \prime} \mathrm{E}\right)$, a ca. 1.5 ha $\left(15000 \mathrm{~m}^{2}\right)$ grass field site approximately $40 \mathrm{~km}$ to the north-east of central London. Measurements of ambient concentrations of a variety of species and meteorological parameters were made by six UK research institutes. Of particular significance to the present investigation were the aerosol mass spectrometric measurements of organic aerosol loading and composition, made by the University of Manchester (McFiggans et al., 2005). As described in the companion to this paper (Johnson et al., 2006), a variety of meteorological conditions were experienced during the five-week campaign and these included periods with prevailing winds from the West, a persistent period of high-pressure and periods with air arriving from the North. In the companion study, a photochemical trajectory model (PTM) was used to simulate mass concentrations of OA material arriving at the measurement site for nine selected dates and times. These nine case study events were chosen due to the availability of aerosol concentration measurements, due to the significant variation in observed aerosol concentrations between the nine events and due to variations in the origin of the air masses arriving at the selected times (as estimated from calculated $96 \mathrm{~h}$ back trajectories). As outlined in the next section, the PTM incorporated a highly-detailed gas-phase degradation mechanism for a large number of emitted VOC, and this was coupled to a representation of SOA formation in the form of equilibrium partitioning of semi- and non-volatile oxidation products, between the gas-phase and an organic aerosol-phase.
This latter was based on the absorptive partitioning theory of Pankow (1994). As described in detail by Johnson et al. (2006), in order to match simulated OA mass concentrations to the hourly-mean concentrations observed by the University of Manchester during TORCH, it was found necessary, first, to scale all (phase-) partitioning coefficients by a factor of 500 and, second, to assume the presence of a constant background of organic aerosol material. The need for scaling was rationalised in terms of the significant occurrence of association reactions occurring between species absorbed in the growing organic aerosol-phase (such chemistry not being represented in the combined PTM), with a possible additional contribution from aqueous uptake of water soluble organic components: and the "ubiquitous" OA background was attributed to highly-processed, persistent oligomeric material resulting from such in-aerosol chemistry.

The first aim of the present study was to further investigate the formation of OA material, for the atmospheric conditions of the TORCH 2003 campaign, by looking at the detailed chemical composition of the simulated SOA for the original nine case study events, and for selected other events. The second aim was to investigate the sensitivity of the simulated mass of SOA to changes in the rates of emission of, in turn, anthropogenic and biogenic VOC, and to compare the results of this exercise with the detailed chemical composition of the simulated SOA.

\section{Methodology}

\subsection{The Photochemical Trajectory Model}

The PTM employed in the present study has recently been described in more detail by Utembe at al. (2005) and Johnson et al. (2006) and thus a much briefer account is given here. The PTM simulates the chemical development in a well-mixed boundary layer air parcel as it is advected along multi-day trajectories. The air parcel picks up emissions of $\mathrm{NO}_{\mathrm{x}}, \mathrm{CO}, \mathrm{SO}_{2}$, methane, anthropogenic VOC and biogenic VOC, which are processed, in the present investigation, using a highly-detailed description of chemical and photochemical transformations (version 3.1 of the Master Chemical Mechanism) leading to the formation of ozone and other secondary pollutants. UK emissions for methane, anthropogenic VOC, $\mathrm{NO}_{\mathrm{x}}, \mathrm{CO}$ and $\mathrm{SO}_{2}$ are based on the 1998 data defined by the National Atmospheric Emissions Inventory (NAEI) (http: //www.aeat.co.uk/netcen/airqual). Emissions for other European countries are based on figures available from EMEP (http://www.emep.int). The emissions were scaled so that the country totals were appropriate to 2003 , based on interpolation of historical and projected figures available from EMEP (http://www.emep.int) and the NAEI, and reported in AQEG $(2004,2005)$. The representation of biogenic VOC emissions for the EMEP domain was based on the methodology described by Dore et al. (2003). 
Emission of primary organic aerosol (POA) material also had to be accounted for in the model. POA not only contributes directly to the ambient organic aerosol concentration but also contributes to the magnitude of the absorbing medium for gas-to-aerosol transfer processes of semi- and non-volatile oxidation products. In the PTM, emissions of POA were defined, on a mass basis, relative to those of $\mathrm{NO}_{\mathrm{x}}$, according to observed correlations between $\mathrm{NO}_{\mathrm{x}}$ volume mixing ratios and mass concentrations of fine organic particulate material $(\leq 200 \mathrm{~nm})$, at several urban locations (Allan et al., 2003; Alfarra, 2004). As discussed in some detail in the companion to this paper (Johnson et al., 2006), it is recognised that relating POA emissions to those of $\mathrm{NO}_{\mathrm{x}}$ is an approximation. However, it provides a practical method which provides a good representation for the region of study, i.e., one in which anthropogenic combustion sources dominate $\mathrm{NO}_{\mathrm{x}}$ and carbonaceous aerosol emissions, and with road transport being the major source. Support for the adopted methodology is provided by the good agreement between the simulated concentrations of POA, and those observed for a wide variety of conditions in the campaign period (McFiggans et al., 2005), as reported in the companion paper.

\subsection{Representation of gas phase chemistry}

As already mentioned, the gas-phase chemical processing of emitted VOC and intermediate oxidation products was represented by the Master Chemical Mechanism, version 3.1 (MCM v3.1). The MCM v3.1 is a near-explicit mechanism which describes the complete degradation of methane and 124 emitted, non-methane VOC and the associated formation of ozone and other secondary pollutants, as described in detail elsewhere (Jenkin et al., 1997, 2003; Saunders et al., 2003; Bloss et al., 2005). The mechanism contains ca. 14000 reactions, which represent the chemistry of ca. 5000 molecular and free radical species. It therefore represents the formation of a large variety of intermediate products bearing a large variety of oxygen-containing functional groups i.e. carbonyl, nitrate, peroxy nitrate, hydroxyl, hydroperoxy, acid and peracid groups, and multifunctional combinations of these groups.

\subsection{Representation of SOA formation}

Again as described in more detail in the companion to this paper (Johnson et al., 2006), SOA formation was represented in terms of the equilibrium partitioning of semiand non-volatile oxidation products between the gas-phase and a particulate- (aerosol-) phase. Equilibrium, phasepartitioning was thus defined for ca. 2000 molecular species, for which estimated boiling temperatures (Joback and Reid, 1987; Stein and Brown, 1994) are $>450 \mathrm{~K}$.

$K_{p, i}=F_{i, o m} /\left(A_{i} \times M_{o}\right)$

Here, $K_{p, i}$ is the partitioning coefficient $\left(\mathrm{m}^{3} \mu \mathrm{g}^{-1}\right), F_{i, o m}$ is the concentration of species $i$ in the condensed organic- phase, $A_{i}$ is its gas-phase concentration and $M_{o}$ is the total mass concentration of condensed organic material $\left(\mu \mathrm{g} \mathrm{m}^{-3}\right)$. Partitioning coefficients were estimated using the absorptive partitioning theory of Pankow (1994):

$K_{p}=\frac{7.501 \times 10^{-9} R T}{M W_{o m} \zeta p_{L}^{\circ}}$

Here, $R$ is the ideal gas constant $\left(8.314 \mathrm{~J} \mathrm{~K}^{-1} \mathrm{~mol}^{-1}\right), T$ is the temperature $(\mathrm{K}), M W_{o m}$ is the mean molecular weight of the absorbing particulate organic material $\left(\mathrm{g} \mathrm{mol}^{-1}\right), \zeta$ is the activity coefficient of the species in the condensed organicphase (assumed to be unity) and $p_{L}^{\circ}$ is its (probably subcooled) liquid vapour pressure (Torr). Partitioning equilibria were represented dynamically by defining each speciesdependent $K_{p}$ value in terms of an absorption $\left(k_{i n}\right)$ and a desorption $\left(k_{\text {out }}\right)$ rate coefficient, with $K_{p}=k_{\text {in }} / k_{\text {out }}$, using a methodology similar to that of Kamens et al. (1999), as described in detail in the companion to this paper.

\subsection{Trajectories and initialisation}

The PTM was used to simulate the chemical development over a $96 \mathrm{~h}$ period along 11 different trajectories arriving at the Writtle site. The trajectories were obtained from the NOAA on-line trajectory service (http://www.arl.noaa.gov/ ready/hysplit4.html) for the original nine case study events (31 July and 2, 4, 6, 8, 16, 19 and 21 August 2003, arriving at 18:00 h; and 18 August 2003, arriving at 12:00 h - see Johnson et al., 2006) and for two additional case study events (7 and 12 August 2003, arriving at 12:00 h). These latter two events were chosen as the emissions of biogenic VOC, integrated along the entire trajectory, were significantly higher than for any of the original nine case study events both in absolute terms and relative to the integrated emissions of anthropogenic VOC. They were not considered originally owing to the unavailability of organic aerosol measurements. The initial concentrations for the majority of species in the simulations were set to zero. As described by Utembe et al. (2005) the initial concentrations for a limited number of inorganic, carbonyl and light hydrocarbon species were set to realistic tropospheric background concentrations for a relatively clean boundary layer.

\section{Results}

The detailed chemical composition of simulated secondary organic aerosol, for the nine case study events considered in the companion to this paper (Johnson et al., 2006), is presented in Table 1. Results are also presented for two additional four-day air trajectories which PTM simulations suggest would have experienced significantly greater emissions of VOC of biogenic origin, in comparison to anthropogenic VOC. 
Table 1. Distributions of species comprising $90 \%$ of simulated SOA mass for nine case study events and for two additional air trajectories (see text). Unless otherwise indicated, numbers correspond to per cent contribution to the total simulated mass of semi- and non-volatile oxidation products partitioned, as monomers, to the aerosol-phase.

\begin{tabular}{|c|c|c|c|c|c|c|c|c|c|c|c|c|c|c|c|c|c|c|c|c|c|c|c|}
\hline & 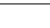 & & & & & rajector & & & & & & & & & & & & rajector & & & & & \\
\hline Species $^{\mathrm{b}}$ & A & В & $\mathrm{C}$ & D & E & F & G & $\mathrm{H}$ & I & $\mathrm{J}^{\mathrm{c}}$ & $\mathrm{K}^{\mathrm{c}}$ & Species $^{b}$ & A & B & C & D & E & F & G & $\mathrm{H}$ & I & $\mathrm{J}^{\mathrm{c}}$ & $\mathrm{K}^{\mathrm{c}}$ \\
\hline C3PAN1 & & & & & & & & & & 0.16 & & C920CO3H & & & & & & 0.23 & & & & & \\
\hline $\mathrm{CO} 23 \mathrm{C} 4 \mathrm{CO} 3 \mathrm{H}$ & & & & & & & & & & 0.37 & 0.32 & HOPINONIC & & & & & & 0.27 & & & & 0.29 & 0.37 \\
\hline C5PAN9 & & & & 0.20 & & & & & & 0.48 & 0.27 & $\mathrm{C} 921 \mathrm{OOH}$ & & & & & & 0.50 & & & & 0.23 & 0.28 \\
\hline $\mathrm{HO} 3 \mathrm{CO} 6 \mathrm{C} 8$ & & & & 0.18 & & & & & & & & $\mathrm{C} 922 \mathrm{OOH}$ & & & & & & 0.42 & & & & 0.22 & 0.28 \\
\hline $\mathrm{C} 82 \mathrm{NO} 3$ & & & & 0.21 & & & & & & & & H1C 23 C 4 PAN & & & & 0.14 & 0.18 & 0.17 & & & & 0.19 & 0.26 \\
\hline $\mathrm{HO} 4 \mathrm{CO} 7 \mathrm{C} 9$ & & & & 0.37 & & & & & & & & C719NO3 & & & & & 0.30 & 0.37 & 0.21 & 0.16 & & & \\
\hline $\mathrm{HO} 3 \mathrm{C} 106 \mathrm{NO} 3$ & & 0.23 & & 0.25 & & & 0.36 & & 0.16 & & & H3C 25 C 6 PAN & 0.56 & 0.70 & 0.62 & 0.45 & 1.07 & 1.23 & 0.80 & 0.65 & 0.74 & 0.22 & 0.44 \\
\hline C8PAN1 & 0.21 & 0.26 & 0.18 & 1.07 & & & & & & 0.22 & 0.13 & H3C2C4PAN & & & & 0.14 & 0.17 & 0.22 & & & & 0.15 & \\
\hline $\mathrm{HO} 5 \mathrm{CO} 8 \mathrm{C} 10$ & 0.36 & 0.61 & & 0.34 & 0.28 & 0.23 & 1.02 & 0.32 & 0.44 & 0.17 & & BPINANO3 & 2.29 & 3.91 & 2.77 & 5.86 & 6.31 & 3.87 & 4.96 & 3.52 & 3.01 & 10.56 & 9.97 \\
\hline C103NO3 & 0.27 & 0.41 & 0.13 & 0.53 & 0.23 & & & 0.17 & & & & BPINAOOH & & & 0.69 & 0.14 & 0.18 & 2.62 & 0.18 & 0.27 & 0.17 & 1.51 & 1.07 \\
\hline $\mathrm{HO} 3 \mathrm{C} 116 \mathrm{NO} 3$ & 0.23 & 0.35 & 0.27 & 0.36 & 0.17 & 0.16 & 0.69 & 0.22 & 0.30 & & & NOPINANO3 & & & & & & & & & & 0.39 & 0.22 \\
\hline C9PAN1 & 0.37 & 0.46 & & 1.79 & 0.24 & & & 0.20 & 0.16 & 0.21 & 0.15 & C9DC & & & & & & & & & & 0.49 & 0.25 \\
\hline $\mathrm{HO} C \mathrm{CO} 9 \mathrm{C} 11$ & 0.75 & 1.14 & 0.15 & 0.34 & 0.55 & 0.54 & 2.30 & 0.71 & 0.99 & 0.20 & 0.15 & C9DCNO3 & & & & & & & & & & 0.60 & 0.53 \\
\hline $\mathrm{C} 113 \mathrm{NO} 3$ & 0.33 & 0.47 & 0.13 & 0.35 & 0.29 & & 0.19 & 0.22 & 0.19 & & & C9DCOOH & & & & & & & & & & 0.14 & 0.19 \\
\hline $\mathrm{C} 113 \mathrm{CO}$ & 0.17 & 0.18 & & & & & & & & & & C9DCCO & & & & & & & & & & 1.36 & 1.28 \\
\hline $\mathrm{HO} 3 \mathrm{C} 126 \mathrm{NO} 3$ & 0.20 & 0.27 & & 0.24 & & 0.15 & 0.63 & 0.20 & 0.29 & & & $\mathrm{C} 914 \mathrm{OOH}$ & & & & & & & & & & 1.00 & 0.20 \\
\hline C10PAN1 & 0.32 & 0.37 & 0.19 & 1.33 & 0.21 & & & 0.18 & & & & C87PAN & & & & & & & & & & 0.53 & 0.49 \\
\hline $\mathrm{HO} 7 \mathrm{CO} 10 \mathrm{C} 12$ & 0.77 & 1.01 & 0.14 & 0.18 & 0.53 & 0.62 & 2.43 & 0.78 & 1.12 & & & $\mathrm{C916OOH}$ & & & & & & & & & & & 0.12 \\
\hline $\mathrm{C} 123 \mathrm{NO} 3$ & 0.20 & 0.27 & & 0.43 & 0.18 & & & & & & & C88PAN & & & & 0.13 & & 0.18 & & & & 0.98 & 0.72 \\
\hline C4PAN6 & 0.21 & 0.30 & 0.15 & 0.14 & & & & & & 0.23 & 0.13 & $\mathrm{C} 88 \mathrm{CO} 3 \mathrm{H}$ & & & & 0.15 & & 0.10 & & & & 0.70 & 0.16 \\
\hline $\mathrm{C} 59 \mathrm{OOH}$ & & & & & & 0.33 & & & & & & $\mathrm{C} 88 \mathrm{CO} 2 \mathrm{H}$ & & & & & & & & & & 0.12 & 0.19 \\
\hline APINANO3 & 1.29 & 2.05 & 1.36 & 2.82 & 3.36 & 2.07 & 2.56 & 1.87 & 1.66 & 3.96 & 3.38 & $\mathrm{C} 88 \mathrm{CO}$ & & & & & & & & & & 0.43 & 0.27 \\
\hline APINAOOH & & & 0.30 & & & 0.80 & & & & 0.36 & 0.24 & C917NO3 & & & & & & & & & & 0.20 & 0.12 \\
\hline PINAL & & & & 0.12 & & & 0.21 & & & 0.10 & 0.06 & NOPINCOOH & & & & & & & & & & 0.30 & 0.15 \\
\hline C96NO3 & & & & & & & & & & 0.35 & 0.20 & $\mathrm{C} 9170 \mathrm{OH}$ & & & & & & 0.21 & & & & 0.35 & 0.39 \\
\hline C98NO3 & 0.99 & 0.97 & 0.94 & 1.14 & 2.31 & 1.71 & 2.49 & 1.59 & 1.56 & 0.65 & 1.27 & $\mathrm{C} 619 \mathrm{CO}$ & & & & & & & & & & 0.22 & 0.13 \\
\hline C614NO3 & & & & 0.20 & 0.23 & 0.15 & 0.34 & 0.19 & 0.20 & 0.14 & 0.15 & BPINBNO3 & & & & 0.25 & 0.25 & & 0.22 & & & 0.62 & 0.29 \\
\hline C10PAN2 & 0.54 & 0.84 & 0.64 & 1.26 & 0.98 & 0.76 & 0.78 & 0.64 & 0.56 & 2.77 & 1.76 & C918PAN & & & 0.13 & & 0.17 & 0.41 & & & & 0.61 & 0.47 \\
\hline PERPINONIC & & & 0.28 & & & 1.06 & & & & 0.97 & 1.10 & NBPINAOOH & & & & & & & & & & 0.66 & 0.22 \\
\hline $\begin{array}{l}\text { PINONIC } \\
\text { late }\end{array}$ & 2.50 & 3.97 & 3.40 & 4.43 & 6.38 & 6.34 & 6.48 & 4.99 & 4.96 & 8.51 & 9.99 & NOPINDCO & & & & & & & & & & 0.17 & \\
\hline $\mathrm{C} 96 \mathrm{OOH}$ & & & & & & & & & & 0.39 & 0.16 & C89PAN & & & & 0.18 & & & & & & 0.37 & 0.17 \\
\hline $\mathrm{C} 96 \mathrm{OH}$ & & & & & & & & & & 0.12 & & С9180OH & & & & & & & & & & 0.16 & 0.14 \\
\hline $\mathrm{C} 97 \mathrm{OOH}$ & 0.22 & & 1.18 & 0.36 & 0.51 & 3.28 & 0.24 & 0.34 & 0.24 & 5.86 & 6.11 & С919OOH & & & 0.16 & & & & & & & 0.18 & 0.25 \\
\hline $\mathrm{C} 98 \mathrm{OOH}$ & & & 0.91 & & 0.16 & 0.51 & & & & 0.52 & 0.95 & C813NO3 & 0.21 & 0.25 & 0.17 & 0.22 & 0.64 & 0.63 & 0.72 & 0.46 & 0.42 & 0.13 & 0.26 \\
\hline $\mathrm{C} 614 \mathrm{OOH}$ & & & & & & & & & & 0.15 & 0.17 & $\mathrm{C} 811 \mathrm{CO} 3 \mathrm{H}$ & & & & & & 1.02 & & & & 0.23 & 0.37 \\
\hline $\mathrm{C} 614 \mathrm{CO}$ & 0.59 & 0.85 & 1.49 & 2.46 & 2.02 & 1.55 & 0.62 & 0.77 & 0.46 & 7.68 & 8.43 & $\begin{array}{l}\text { PINIC } \\
\text { PIN }\end{array}$ & & & & & & 1.08 & & & & 0.77 & 1.08 \\
\hline PINALOOH & & & 0.20 & & & 0.25 & & & & 0.24 & 0.24 & C81IPAN & 2.83 & 3.13 & 2.59 & 2.53 & 4.98 & 5.41 & 5.29 & 5.43 & 5.40 & 2.31 & 3.34 \\
\hline $\mathrm{C} 106 \mathrm{OOH}$ & & & 0.24 & & & & & & & & 0.16 & $\mathrm{C} 811 \mathrm{OOH}$ & & & & & & 0.25 & & & & 0.32 & 0.30 \\
\hline $\mathrm{C} 716 \mathrm{OH}$ & 0.16 & & 0.16 & 0.16 & 0.23 & & 0.58 & 0.27 & 0.25 & & & C721CHO & & & & & & 0.28 & & & & 0.67 & 0.64 \\
\hline C9PAN2 & & & & & & & & & & 0.83 & 0.41 & $\mathrm{C} 812 \mathrm{OOH}$ & & & 0.24 & & & 1.70 & & & & 0.95 & 1.59 \\
\hline $\mathrm{C} 85 \mathrm{CO} 3 \mathrm{H}$ & & & & & & & & & & 0.49 & 0.49 & $\mathrm{C} 813 \mathrm{OOH}$ & & & 0.13 & 0.21 & 0.21 & 0.24 & & & & & 0.20 \\
\hline $\mathrm{C} 85 \mathrm{OOH}$ & & & & & & & & & & 0.21 & & C721PAN & 0.27 & 0.39 & 0.61 & 0.38 & 0.68 & 1.68 & & 0.53 & 0.19 & 2.21 & 3.19 \\
\hline $\mathrm{C} 86 \mathrm{OOH}$ & & & & & & & & & & 0.19 & & $\mathrm{C} 721 \mathrm{CO} 3 \mathrm{H}$ & & & & & & & & & & 0.16 & 0.32 \\
\hline C7PAN3 & & & 0.17 & 0.21 & 0.18 & 0.22 & & & & 0.57 & 0.36 & NORPINIC & & & & & & & & & & 0.25 & 0.43 \\
\hline APINBNO3 & 0.58 & 0.97 & 0.67 & 1.59 & 1.58 & 0.64 & 1.07 & 0.80 & 0.71 & 2.24 & 1.75 & $\mathrm{C} 721 \mathrm{OOH}$ & & & & & & & & & & 0.58 & $\begin{array}{l}0.52 \\
0.52\end{array}$ \\
\hline APINBCO & & 0.20 & 0.32 & 0.56 & 0.44 & 0.95 & & 0.25 & & 1.81 & 1.31 & $\mathrm{C} 722 \mathrm{OOH}$ & & & & & & & & & & 0.19 & 0.25 \\
\hline NAPINAOOH & & & & & & & 0.20 & & & 1.67 & 0.56 & C811NO3 & & 0.41 & 0.27 & 0.71 & 0.73 & 0.38 & 0.42 & 0.25 & 0.16 & 2.37 & 2.82 \\
\hline NAPINBOOH & & & & & & & & & & 0.17 & & C23O3CPAN & 0.32 & 0.45 & 0.16 & 0.53 & 0.19 & & & 0.18 & & 0.21 & \\
\hline $\mathrm{NC} 1010 \mathrm{OH}$ & & & & & & & & & & 0.32 & 0.29 & C2303ECPAN & 0.15 & 0.33 & & 0.51 & & & & & & & \\
\hline $\mathrm{NCl} 102 \mathrm{OOH}$ & & & & & & & & & & 0.29 & 0.35 & C2303MCPAN & 0.18 & 0.36 & & 0.53 & & & & & & & \\
\hline C108NO3 & & & & & & & & & & & & DNCRES & 1.16 & 1.02 & 3.68 & 1.42 & 1.42 & 0.22 & 0.57 & 1.03 & 0.91 & 0.38 & 0.51 \\
\hline $\mathrm{ClO7OOH}$ & & & 0.23 & & & 0.15 & & & & 0.25 & & DNEBNZOL & & 0.17 & 0.58 & 0.28 & & & & & & & \\
\hline $\mathrm{C} 108 \mathrm{OOH}$ & & & 0.29 & & & & & & & & & DNMETOL & & & 0.28 & 0.17 & & & & & & & \\
\hline CO3H4PAN & & & & 0.17 & & & & & & & & DNMXYOL & 0.52 & 0.66 & 1.61 & 0.94 & 0.59 & & & 0.34 & 0.25 & 0.25 & 0.27 \\
\hline TLCOBIPEOH & & & & & & & 0.17 & & & & & DNOETOL & & & 0.14 & & & & & & & & \\
\hline MALANHY & 12.18 & 14.38 & 12.33 & 13.56 & 10.45 & 7.17 & 9.46 & 10.13 & 9.79 & 4.23 & 4.72 & DNOXYOL & 0.39 & 0.36 & 0.99 & 0.46 & 0.46 & & 0.18 & 0.36 & 0.28 & 0.12 & 0.16 \\
\hline MALANHYOOH & & & 0.14 & & & & & & & & & DNPETOL & & & 0.15 & & & & & & & & \\
\hline MALNHYOHCO & 0.34 & & 0.64 & & 0.42 & 0.90 & 0.38 & 0.51 & 0.94 & 0.17 & 0.28 & DNPHEN & 1.36 & 1.62 & 6.37 & 2.11 & 1.92 & & 0.47 & 0.85 & 0.58 & 0.45 & 0.53 \\
\hline TLBIPERNO3 & & 0.18 & & 0.31 & & & 0.52 & & 0.22 & & & DNPXYOL & 0.26 & 0.31 & 0.81 & 0.45 & 0.30 & & & 0.18 & & 0.12 & 0.14 \\
\hline
\end{tabular}

As described and discussed by Johnson et al. (2006), a "ubiquitous" OA background concentration of $0.7 \mu \mathrm{g} \mathrm{m}^{-3}$ was also inferred from the analysis and observations, and attributed to the presence of highly-processed, persistent, oligomeric aerosol material which is formed over long timescales. The mass concentrations of primary and secondary organic aerosol material (as listed towards the foot of the table) are as reported by Johnson et al. (2006), and the detailed speciation of the SOA component is presented in terms of per cent $(\%)$ contributions of individual oxidation products to the simulated total mass concentration of oxidation products partitioned to the aerosol-phase. Also presented in Table 1 are the fractional (per cent) contributions of the simulated SOA mass which are made up of species containing a selection of oxygenated functional groups, namely aldehyde, ketone, ester, anhydride, hydroxyl, hydroperoxy, acid, nitro, nitrate and peroxynitrate groups.

The chemical nomenclature used in Table 1 is that of the MCM, and the structures of the twenty most significant SOA- mass-contributing species, for each of the original nine case study events, are collectively indicated in Fig. 1. In each case, these species represent about $70 \%$ of the total simulated SOA mass (i.e. the summed mass of "monomers" partitioned to the aerosol-phase). As a first diagnosis, Table 2 represents the data in Table 1 by categorising each of the species in terms of the primary, emitted, VOC from which they are predominantly derived in the MCM v3.1 and summing the per cent contributions to SOA mass for each of these categories. It should be noted that certain of the species predicted to contribute to the SOA mass may be derived from more than one emitted VOC precursor. Most significantly for the present investigation, in the MCM v3.1, maleic anhydride (furan-2,5-dione) may be formed from the photooxidation of benzene, toluene, ethylbenzene, $o$-xylene, $m$-xylene, $n$-propylbenzene, $i$-propylbenzene, $o$-ethyltoluene and $m$ ethyltoluene. That said, in the present simulations, the majority of maleic anhydride is expected to be derived from toluene (due to a combination of the yield of butenedial - 
Table 1. Continued.

\begin{tabular}{|c|c|c|c|c|c|c|c|c|c|c|c|c|c|c|c|c|c|c|c|c|c|c|c|}
\hline TLOBIPEROH & 0.17 & 0.24 & & 0.44 & & 0.17 & 0.53 & 0.17 & 0.22 & & & EMALANHY & & & 0.25 & 0.16 & & & & & & & \\
\hline MMALANHY & 1.10 & 1.57 & $\begin{array}{l}1.69 \\
0.26\end{array}$ & 2.49 & 1.11 & 0.42 & 1.26 & 0.79 & 0.80 & 0.99 & 0.68 & $\begin{array}{l}\text { ENCATECH } \\
\end{array}$ & 2.26 & 1.68 & 0.67 & 0.23 & 1.43 & 1.14 & 1.12 & 1.77 & 1.58 & & \\
\hline $\begin{array}{l}\text { TLFUOOH } \\
\text { TLOH }\end{array}$ & & & & & & 0.19 & & & & & & ENNCATCOOH & & & 0.15 & & & & & & & & \\
\hline MXYOBPEROH & 0.19 & & & 0.42 & & 0.22 & 0.96 & 0.22 & 0.34 & & & IPNCATECH & 0.22 & & & & & & & 0.17 & 0.16 & & \\
\hline MXYBIPENO3 & 0.16 & & & 0.26 & & 0.14 & 0.94 & 0.19 & 0.35 & & & MNCATECH & 8.77 & 6.16 & 2.63 & 0.82 & 5.05 & 7.35 & 3.96 & 7.98 & 7.18 & 0.14 & 0.41 \\
\hline MXYMUCNO3 & 0.67 & 0.53 & & 1.52 & 0.33 & 0.73 & 1.56 & 0.75 & 1.11 & & & MNCATECOOH & 0.55 & & 0.87 & & 0.42 & 0.67 & 0.49 & 0.37 & 0.96 & & \\
\hline MXYMUCCO & & & & 0.37 & & 0.18 & 0.22 & & 0.17 & & & MNNCATCOOH & 0.21 & & 0.68 & 0.18 & 0.28 & & 0.26 & & $\begin{array}{l}0.19 \\
0.19\end{array}$ & 0.21 & 0.44 \\
\hline HOC6H4NO2 & 0.77 & 1.22 & 0.97 & 1.85 & 0.77 & 0.18 & 0.63 & 0.52 & 0.48 & 0.42 & 0.29 & MTNCATECH & 0.55 & 0.39 & & & 0.33 & 0.48 & 0.59 & 0.53 & 0.55 & & \\
\hline TOL1OHNO2 & 0.73 & 0.95 & 0.75 & 1.59 & 0.57 & 0.41 & 0.38 & 0.63 & 0.63 & 0.48 & 0.39 & MXNCATCOOH & & & 0.16 & & & 0.17 & & & 0.21 & & \\
\hline MXY1OHNO2 & 0.80 & 1.17 & 0.55 & 1.90 & 0.67 & 0.39 & 0.95 & 0.67 & 0.80 & 0.23 & 0.24 & MXNCATECH & 2.96 & 2.12 & 0.42 & 0.25 & 1.75 & 3.29 & 3.85 & 3.13 & 3.37 & & \\
\hline TOL4OHNO2 & & & 0.15 & & & & & & & & & MXNNCATOOH & & & 0.12 & & & & & & & & \\
\hline PXYBIPENO3 & & & & 0.24 & & & 0.65 & & 0.25 & & & NBZFUONE & & & & 0.15 & & & & & & 0.18 & \\
\hline PXY1OHNO2 & 0.39 & 0.59 & 0.30 & 0.89 & 0.34 & 0.18 & 0.34 & 0.32 & 0.35 & 0.13 & 0.13 & $\mathrm{NC} 4 \mathrm{DCO} 2 \mathrm{H}$ & 1.68 & 1.41 & 3.82 & 2.02 & 1.77 & 0.23 & 0.72 & 1.39 & 1.33 & 0.22 & 0.32 \\
\hline OXYBIPENO3 & & & & 0.15 & & & 0.40 & & 0.15 & & & $\mathrm{NC} 4 \mathrm{EDCO} 2 \mathrm{H}$ & 1.78 & $\begin{array}{l}1.41 \\
1.65\end{array}$ & $\begin{array}{l}.02 \\
1.95\end{array}$ & 1.33 & 1.33 & 0.24 & 0.22 & 1.07 & 0.81 & 0.20 & 0.33 \\
\hline OXY1OHNO2 & 0.48 & 0.60 & 0.33 & 0.85 & 0.36 & 0.34 & 0.37 & 0.47 & 0.48 & 0.14 & 0.15 & NC4IPDCO2H & 0.18 & & 0.20 & 0.13 & & & & & & & \\
\hline TM135BPNO3 & & & & & & & 0.62 & & 0.26 & & & $\mathrm{NC} 4 \mathrm{MDCO} 2 \mathrm{H}$ & $\begin{array}{l}15.04 \\
150\end{array}$ & 13.68 & 12.20 & 9.67 & 10.02 & 5.47 & 4.18 & 11.88 & 10.81 & 1.44 & 2.40 \\
\hline TM135OBPOH & & & & & & & 0.51 & & 0.17 & & & NC4PDCO2H & 0.33 & 0.29 & 0.39 & 0.23 & 0.25 & & & 0.20 & 0.15 & & \\
\hline DM124OHNO2 & & & 0.13 & & & & & & & & & $\mathrm{NC} 5 \mathrm{MDCO} 2 \mathrm{H}$ & 0.21 & 0.24 & 0.13 & 0.17 & 0.16 & & & & & & \\
\hline TM124BPNO3 & 0.16 & & & 0.18 & & 0.14 & 0.95 & 0.19 & 0.36 & & & NCATECHOL & 5.69 & 3.29 & 4.05 & 0.93 & 3.97 & 2.30 & 1.60 & 5.27 & 5.18 & 0.12 & 0.34 \\
\hline TMBIFUOOH & & & 0.16 & & & & & & & & & NCATECOOH & & & 0.49 & & 0.24 & & 0.18 & & 0.21 & & \\
\hline TM124MUNO3 & & & & & & & 0.23 & & & & & NCRES1OOH & & & 0.26 & & & & & & & 0.29 & 0.26 \\
\hline TM123BPNO3 & & & & & & & 0.25 & & & & & NEBFUONE & & & & 0.33 & & & & & & & \\
\hline TM124OHNO2 & & & & 0.17 & & & & & & & & NMXYOLIOOH & & & 0.22 & & & & & & & 0.21 & 0.21 \\
\hline EBZBPERNO3 & & & & & & & 0.26 & & & & & NNCATECOOH & & & 0.48 & 0.12 & 0.17 & & & & & 0.21 & 0.21 \\
\hline EBNZOHNO2 & 0.23 & 0.36 & 0.25 & 0.55 & 0.22 & & & & & & & NOXYOL1OOH & & & 0.14 & & & & & & & & 0.13 \\
\hline OET1OHNO2 & & & & 0.19 & & & & & & & & NPHEN1OOH & & & 0.17 & & & & & & & 0.18 & \\
\hline METLBIPNO3 & & & & & & & 0.32 & & & & & OTNCATECH & 0.54 & 0.39 & & & 0.34 & 0.39 & 0.44 & 0.48 & 0.47 & & \\
\hline METLOBPROH & 0.60 & 0.77 & 0.71 & 0.78 & 0.52 & 0.22 & 0.44 & 0.41 & 0.39 & 0.18 & 0.20 & OXNCATCOOH & 0.33 & 0.39 & 0.20 & & 0.34 & 0.49 & 0.26 & 0.48 & 0.57 & & \\
\hline METLMUCNO3 & & & & & & & 0.22 & & & & & OXNCATECH & 3.02 & 2.04 & 0.51 & 0.23 & 1.66 & 4.64 & 2.51 & 3.52 & 3.41 & & \\
\hline MET1OHNO2 & 0.23 & 0.31 & 0.16 & 0.41 & 0.20 & 0.31 & 0.23 & 0.18 & 0.21 & & & OXNNCATOOH & & & 0.15 & & & & & & & & \\
\hline PETLBIPNO3 & & & & & & & 0.24 & & & & & $\begin{array}{l}\text { PNCATECH } \\
\text { PNT }\end{array}$ & 0.41 & 0.29 & 0.13 & & 0.25 & 0.20 & 0.18 & 0.32 & 0.28 & & \\
\hline PET1OHNO2 & & & & 0.20 & & & & & & & & PTNCATECH & 0.56 & 0.40 & & & & 0.40 & 0.45 & 0.50 & 0.49 & & \\
\hline DMEBIPNO3 & 0.41 & & & 0.15 & & & 2.27 & 0.50 & 1.05 & & & PXNCATCOOH & & & 0.18 & & & 0.16 & & & 0.22 & & \\
\hline DMEBMUNO3 & & & & & & & 0.18 & & & & & PXNCATECH & 2.78 & 2.00 & 0.50 & 0.23 & & 2.73 & 2.49 & 2.74 & 2.72 & & \\
\hline DETLBIPNO3 & & & & & & & 0.53 & & 0.27 & & & $\begin{array}{l}\text { PXNNCATOOH } \\
\text { PXNOF }\end{array}$ & 2.10 & 2.00 & 0.13 & 0.25 & & 2.75 & 2.49 & 2.14 & 2.12 & & \\
\hline $\mathrm{C} 109 \mathrm{OOH}$ & & & 0.19 & & & & & & & & & T123NCATEC & & & & & & 0.14 & 0.20 & & 0.16 & & \\
\hline \multirow[t]{2}{*}{ C920PAN } & 1.33 & 0.99 & 1.20 & 0.84 & 1.44 & 1.01 & 1.97 & 2.80 & 3.23 & 0.47 & 0.72 & T124NCATEC & 0.15 & & & & & 0.16 & 0.23 & 0.16 & 0.18 & & \\
\hline & & & & & & & & & & & & \multicolumn{12}{|c|}{ Per cent contribution of monomer species containing the following groups ${ }^{i}$ : } \\
\hline $\mathrm{E}_{\mathrm{AVOC}}^{\mathrm{d}}$ & 234 & 496 & 349 & 1102 & 189 & 96.0 & 507 & 138 & 195 & 786 & 365 & aldehyde & 19.8 & 17.6 & 20.0 & 15.7 & 13.9 & 7.5 & 8.3 & 15.6 & 14.6 & 4.6 & 5.7 \\
\hline $\begin{array}{l}\text { EVOC } \\
\mathrm{E}_{\mathrm{BVOC}}^{\mathrm{d}}\end{array}$ & 9.08 & 21.8 & 23.4 & 102 & 28.4 & 12.2 & 14.7 & 10.8 & 8.26 & 566 & 334 & ketone & 13.0 & 16.1 & 15.4 & 22.2 & 21.1 & 26.8 & 26.3 & 17.6 & 18.5 & 44.0 & 43.9 \\
\hline $\mathrm{E}_{\mathrm{NO}_{\mathrm{x}}}^{\mathrm{d}}$ & 243 & 403 & 323 & 941 & 306 & 80.2 & 354 & 214 & 240 & 597 & 286 & ester ${ }^{j}$ & 0.7 & 1.1 & 0.3 & 2.0 & 0.2 & 0.2 & 0.0 & 0.2 & 0.0 & 0.4 & 0.0 \\
\hline $\begin{array}{l}\mathrm{N}_{\mathrm{NO}} \\
\mathrm{E}_{\mathrm{BVOC}} / \mathrm{E}_{\mathrm{AVOC}}\end{array}$ & 0.039 & 0.044 & 0.067 & 0.093 & 0.15 & 0.13 & 0.029 & 0.079 & 0.042 & 0.72 & 0.92 & anhydride $^{k}$ & 13.6 & 16.0 & 15.3 & 16.2 & 12.0 & 8.5 & 11.1 & 11.4 & 11.5 & 5.4 & 5.7 \\
\hline $\mathrm{E}_{\mathrm{VOC}} / \mathrm{E}_{\mathrm{NO}_{\mathrm{x}}}$ & 1.00 & 1.28 & 1.15 & 1.28 & 0.71 & 1.35 & 1.47 & 0.70 & 0.85 & 2.26 & 2.44 & hydroxy & 51.4 & 46.8 & 47.3 & 47.9 & 48.8 & 56.6 & 60.1 & 52.1 & 55.2 & 44.2 & 44.7 \\
\hline$[\mathrm{POA}] / \mu \mathrm{g} \mathrm{m}^{-3 \mathrm{e}}$ & 0.33 & 0.63 & 0.41 & 1.25 & 0.45 & 0.07 & 0.61 & 0.22 & 0.26 & 0.76 & 0.42 & hydroperoxy & 1.3 & 0.0 & 10.2 & 1.0 & 2.2 & 15.3 & 1.8 & 1.2 & 2.8 & 19.5 & 19.2 \\
\hline$[\mathrm{SOA}] / \mu \mathrm{g} \mathrm{m}^{-3 \mathrm{f}}$ & 0.51 & 1.63 & 1.08 & 5.01 & 1.11 & 0.22 & 0.79 & 0.29 & 0.23 & 19.3 & 4.58 & acid & 25.0 & 25.4 & 26.1 & 22.0 & 27.2 & 25.2 & 18.0 & 26.2 & 24.2 & 21.9 & 28.9 \\
\hline$[\mathrm{OA}]_{\text {total }} / \mu \mathrm{g} \mathrm{m}^{-3 \mathrm{~g}}$ & 1.54 & 2.96 & 2.19 & 6.96 & 2.26 & 0.99 & 2.10 & 1.21 & 1.19 & 20.8 & 5.70 & nitro & 55.5 & 45.4 & 50.4 & 31.0 & 37.2 & 32.7 & 28.0 & 47.2 & 46.2 & 5.7 & 7.7 \\
\hline$[\mathrm{OA}]_{\mathrm{obs}} / \mu \mathrm{g} \mathrm{m}^{-3 \mathrm{~h}}$ & 1.91 & 3.64 & 4.02 & 6.92 & 5.52 & 0.84 & 2.78 & 1.24 & 0.93 & - & - & nitrate & 8.2 & 11.3 & 8.4 & 18.8 & 17.4 & 11.1 & 25.5 & 11.3 & 12.9 & 25.7 & 22.8 \\
\hline & & & & & & & & & & & & peroxynitrate & 7.3 & 8.6 & 6.6 & 12.5 & 10.5 & 11.3 & 8.8 & 10.6 & 10.3 & 13.7 & 13.0 \\
\hline
\end{tabular}

${ }^{\mathrm{a}}$ Letters correspond to the following trajectory arrival dates and times: A - 31/07/03, 18:00; B - 02/08/03, 18:00; C - 04/08/03, 18:00; D 06/08/03, 18:00; E - 08/08/03, 18:00; F - 16/08/03, 18:00; G - 18/08/03, 12:00; H - 19/08/03, 18:00; I - 21/08/03, 18:00;

${ }^{b}$ MCM name (consult MCM website to find corresponding structures, some of which are also given in Fig. 1);

$\mathrm{c}$ additional trajectory arrival dates and times having experienced relatively greater biogenic VOC emissions (see text), $\mathrm{J}-07 / 08 / 03,12: 00$; K - 12/08/03, 12:00;

d mean mass emission rates of anthropogenic VOC $\left(\mathrm{E}_{\mathrm{VOC}}\right)$, biogenic $\mathrm{VOC}\left(\mathrm{E}_{\mathrm{BVOC}}\right)$ and $\mathrm{NO}_{\mathrm{x}}\left(\mathrm{E}_{\mathrm{NO}_{\mathrm{x}}}\right.$; as $\left.\mathrm{NO}_{2}\right)$ during $96 \mathrm{~h}$ simulation period, in units of $\mu \mathrm{g} \mathrm{m}^{-2} \mathrm{hr}^{-1}$;

e simulated mass concentration of primary organic aerosol;

$\mathrm{f}$ simulated mass concentration of secondary organic aerosol (i.e. the mass sum of all the species listed in the first column of this table partitioned to the aerosol-phase);

$\mathrm{g}$ simulated total mass of organic aerosol material. This is the sum of [POA], [SOA] and a background aerosol concentration of $\mu \mathrm{g} \mathrm{m}^{-3}$;

$\mathrm{h}$ total organic aerosol concentration observed using aerosol mass spectrometry by the University of Manchester;

${ }^{\mathrm{i}}$ fractional contribution of simulated SOA mass (in percent) made up of monomer species containing the given substituent functional group (note that the given species may contain more than one such substitution; and that the majority of species are multifunctional, and therefore usually contain two or more of the listed functional groups);

j "ester" category includes "furanones";

k "anhydride" category includes "furandiones".

from which maleic anhydride is formed - in the toluene degradation mechanism, and the high emissions of toluene relative to the other aromatics) with a smaller contribution from benzene (although the butenedial formation efficiency is relatively larger for benzene, the applied average emission rate of benzene is about one of third of that for toluene). Thus, the maleic anhydride contribution to SOA mass is attributed to toluene and included in the corresponding row of Table 2. For the other cases of species derived from more than one aromatic precursor, their mass contributions to SOA are attributed to the aromatic VOC with the highest emission rate in the PTM. Additional rows are included in the table which indicate the summed contributions of products derived from the photooxidation of $\mathrm{C}_{8}$ and $\mathrm{C}_{9}$ aromatic species. Finally, the last two rows in the table describe the simulated SOA in terms of species being derived from emitted VOC of either anthropogenic or biogenic origin. 


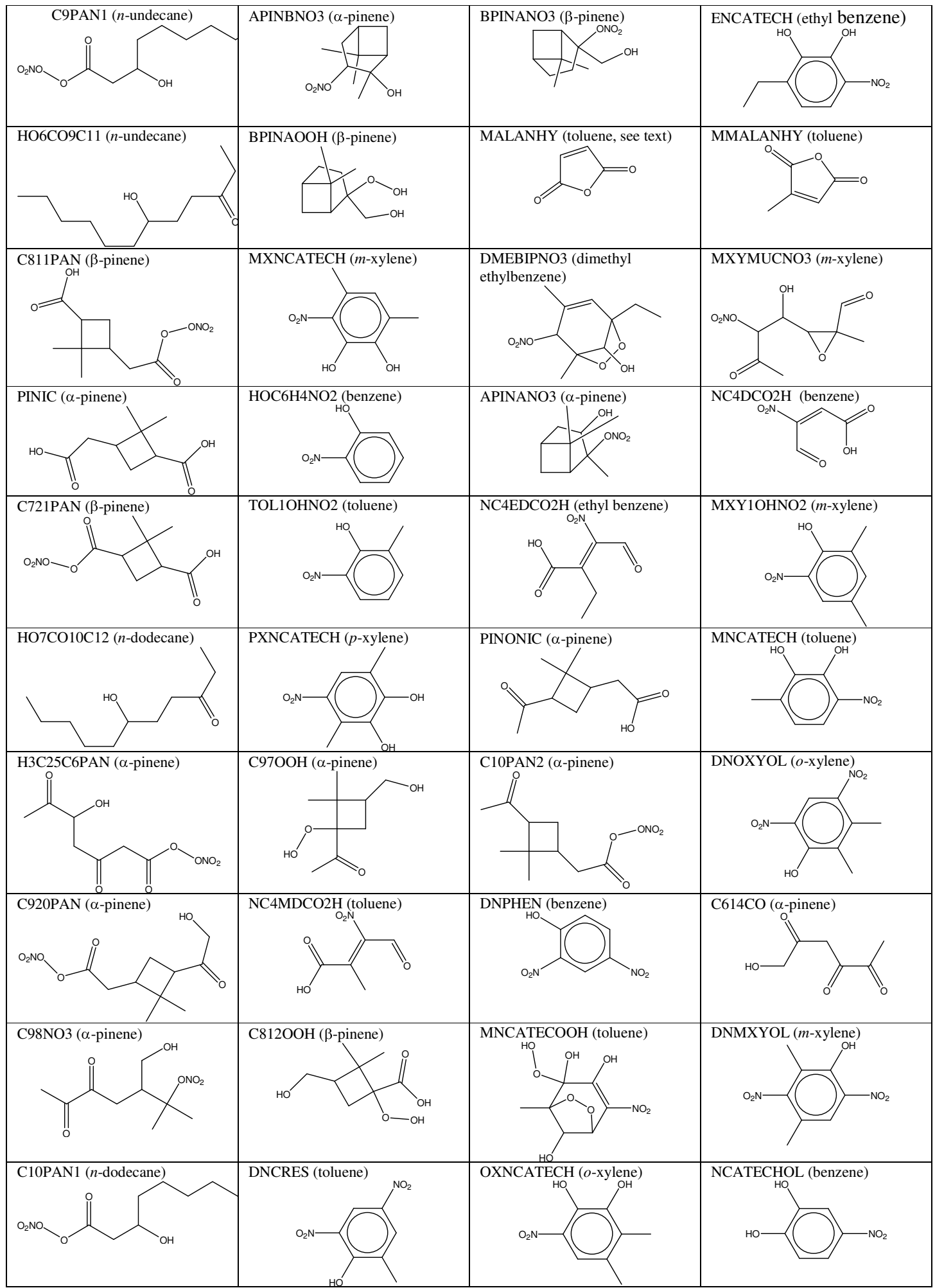

Fig. 1. Structures and MCM names of principal species contributing to simulated SOA mass. The major emitted VOC from which each species is derived in the MCM v3.1 is indicated in parentheses. 
Table 2. Per cent contributions of oxidation products to the mass of simulated SOA, categorised according to (emitted) precursor VOC.

\begin{tabular}{|c|c|c|c|c|c|c|c|c|c|c|c|}
\hline \multirow[b]{2}{*}{ Emitted Precursor Species } & \multicolumn{11}{|c|}{ Trajectory $^{\mathrm{a}}$} \\
\hline & A & $\mathrm{B}$ & $\mathrm{C}$ & $\mathrm{D}$ & $\mathrm{E}$ & $\mathrm{F}$ & G & $\mathrm{H}$ & I & $\mathrm{J}$ & $\mathrm{K}$ \\
\hline$\alpha$-pinene ${ }^{b}$ & 8.76 & 11.6 & 14.8 & 16.9 & 21.5 & 24.9 & 18.6 & 15.3 & 14.6 & 43.5 & 42.2 \\
\hline$\beta$-pinene ${ }^{\mathrm{b}}$ & 5.61 & 8.09 & 7.75 & 10.6 & 14.2 & 20.0 & 11.8 & 10.5 & 9.34 & 32.1 & 33.3 \\
\hline Isoprene & 0.21 & 0.30 & 0.15 & 0.14 & & 0.33 & & & & 0.23 & 0.13 \\
\hline Butane & & & & & & & & & & 0.16 & \\
\hline Hexane & & & & 0.20 & & & & & & 0.85 & 0.59 \\
\hline Octane & & & & 0.39 & & & & & & & \\
\hline Nonane & & & & 0.37 & & & & & & & \\
\hline Decane & 0.83 & 1.51 & 0.31 & 2.20 & 0.51 & 0.23 & 1.38 & 0.49 & 0.59 & 0.39 & 0.13 \\
\hline Undecane & 1.84 & 2.59 & 0.55 & 2.84 & 1.24 & 0.69 & 3.18 & 1.35 & 1.65 & 0.41 & 0.31 \\
\hline Dodecane & 1.49 & 1.92 & 0.33 & 2.18 & 0.91 & 0.77 & 3.06 & 1.17 & 1.40 & & \\
\hline Benzene & 9.50 & 7.55 & 16.4 & 7.18 & 8.83 & 2.72 & 3.60 & 8.03 & 7.77 & 1.57 & 1.48 \\
\hline Toluene & 40.2 & 38.2 & 36.1 & 30.5 & 29.8 & 23.0 & 22.0 & 33.5 & 32.7 & 8.34 & 10.1 \\
\hline$o$-Xylene & 3.84 & 2.64 & 1.32 & 1.24 & 2.02 & 5.47 & 3.71 & 4.20 & 4.61 & 0.14 & 0.27 \\
\hline$m$-Xylene & 5.48 & 4.84 & 3.09 & 6.19 & 3.34 & 5.12 & 8.48 & 5.31 & 6.60 & 0.69 & 0.72 \\
\hline$p$-Xylene & 3.75 & 3.35 & 2.22 & 2.33 & 0.83 & 3.07 & 3.48 & 3.41 & 3.53 & 0.47 & 0.27 \\
\hline Ethyl benzene & 4.27 & 3.87 & 4.06 & 3.06 & 2.98 & 1.38 & 1.60 & 2.83 & 2.39 & 0.20 & 0.33 \\
\hline$n$-Propyl benzene & 0.74 & 0.58 & 0.52 & 0.23 & 0.50 & 0.20 & 0.18 & 0.52 & 0.43 & & \\
\hline$i$-Propyl benzene & 0.40 & & 0.20 & 0.13 & & & & 0.17 & 0.16 & & \\
\hline 1,2,3-Trimethyl benzene & & & & 0.17 & & 0.14 & 0.45 & & 0.16 & & \\
\hline 1,2,4-Trimethyl benzene & 0.52 & 0.24 & 0.29 & 0.35 & 0.16 & 0.30 & 2.03 & 0.35 & 0.81 & & \\
\hline 1,3,5-Trimethyl benzene & & & 0.13 & & & & 0.51 & & 0.17 & & \\
\hline$o$-Ethyl toluene & 0.54 & 0.39 & 0.14 & 0.19 & 0.34 & 0.39 & 0.44 & 0.48 & 0.47 & & \\
\hline$m$-Ethyl toluene & 1.37 & 1.47 & 1.16 & 1.36 & 1.05 & 1.01 & 1.80 & 1.12 & 1.15 & 0.18 & 0.20 \\
\hline$p$-Ethyl toluene & 0.56 & 0.40 & 0.15 & 0.20 & & 0.40 & 0.70 & 0.50 & 0.49 & & \\
\hline 3,5-Dimethyl ethylbenzene & 0.56 & 0.33 & & 0.66 & & & 2.44 & 0.50 & 1.05 & & \\
\hline 3,5-Diethyl toluene & & & & & & & 0.53 & & 0.27 & & \\
\hline$\Sigma\left(\mathrm{C}_{8} \text { Aromatics }\right)^{\mathrm{c}}$ & 17.3 & 14.7 & 10.7 & 12.8 & 9.16 & 15.1 & 17.26 & 15.8 & 17.1 & 1.49 & 1.59 \\
\hline$\Sigma\left(\mathrm{C}_{9} \text { Aromatics }\right)^{\mathrm{d}}$ & 4.14 & 3.07 & 2.57 & 2.63 & 2.05 & 2.43 & 6.12 & 3.14 & 3.84 & 0.18 & 0.20 \\
\hline$\Sigma(\text { Biogenic })^{\mathrm{e}}$ & 16.1 & 22.2 & 25.3 & 30.9 & 40.5 & 50.2 & 33.8 & 28.7 & 26.5 & 85.0 & 84.0 \\
\hline$\Sigma(\text { Anthropogenic })^{\mathrm{f}}$ & 83.9 & $\mathbf{7 7 . 8}$ & 74.7 & 69.2 & 59.5 & 49.8 & 66.2 & 71.3 & 73.5 & 15.0 & 16.0 \\
\hline
\end{tabular}

a Letters correspond to trajectory arrival dates and times as described in the footnote of Table 1;

$\mathrm{b} \alpha$ - and $\beta$-pinene are used to represent all monoterpenes (see Johnson et al., 2006);

${ }^{\mathrm{c}}$ summed contributions of $o-, m$ - and $p$-xylene, and ethyl benzene;

$\mathrm{d}$ summed contributions of $n$ - and $i$-propyl benzene, 1,2,3-, 1,2,4- and 1,3,5-trimethyl benzene, and $o$-, $m$ - and $p$-ethyl toluene;

e summed contributions of $\alpha$ - and $\beta$-pinene, and isoprene;

$\mathrm{f}$ summed contribution of remaining species listed in the first column of this table.

\section{Discussion}

\subsection{SOA composition}

Before discussing the speciation of simulated SOA for selected events during the first TORCH measurement campaign, some discussion of what the data in Tables 1 and 2 represent is required. The results of previous simulations of SOA formation that used the highly-detailed MCM gasphase degradation mechanism, coupled to a representation of gas-to-particle transfer, were unable to reproduce experimental observations without significant scaling of partitioning coefficients for all semi- and non-volatile oxidation products (viz., SOA formation from the ozonolysis of $\alpha$ - and $\beta$-pinene
(Jenkin, 2004) and from the photooxidation of various aromatic hydrocarbons (Johnson et al., 2004, 2005), observed in environmental chamber experiments). As already mentioned and discussed in the companion to this paper (Johnson et al., 2006), scaling of all phase-partitioning coefficients was also found to be necessary in simulating the selected case study events of the 2003 TORCH campaign and, as in the chamber experiment simulations, was attributed to the occurrence of association chemistry in the organic aerosolphase which was not represented in the model. Association, or "accretion", reactions give rise to the production of adduct species (including large molecular weight oligomers) which are less volatile than the reactants from which they are formed. This means that, effectively, the absorbed reactants 
are "stabilised" within the condensed organic-phase with respect to their being in the gas-phase. For the TORCH simulations it was found necessary to increase all partitioning coefficients by a factor of 500 and also, as already mentioned above, to assume the presence of a persistent background concentration of organic aerosol, of $0.7 \mu \mathrm{g} \mathrm{m}^{-3}$, in order to match the concentrations of organic aerosol material observed during the TORCH campaign. Again, this is discussed in greater detail by Johnson et al. (2006). What the data in Tables 1 and 2 represent are simulated distributions (in the form of individual mass contributions to the total simulated mass of SOA) of oxidation products which may potentially participate in association reactions within the aerosol phase - as such, they are referred to as "monomers" in the tables.

It is recognised that applying a single scaling factor to the phase-partitioning coefficients of all organic oxidation products, to account for condensed-phase association and accretion reactions, is a significant simplification. Also recognising that development of an explicit representation of accretion reactions is impractical (even representing simple association reactions for the 2000 species would require 2 million reactions), the possibility of applying species-dependent scaling factors based on the functional group content of the 2000 partitioning species was considered. As discussed further below, a number of published studies have proposed mechanisms for condensed-phase association and accretion reactions (e.g., Tobias and Ziemann, 2000; Jang and Kamens, 2001a, 2001b; Jang et al., 2002; Kalberer et al., 2004; Gao et al., 2005; Liggio et al., 2005; Jang et al., 2005), or have suggested species which are likely to have a greater tendency to participate in such mechanisms (e.g., Barsanti and Pankow, 2004). Although development of a corresponding refined approach is a logical (and indeed proposed) extension to the present work, examination of the species identified in Table 1 and Fig. 1 reveals that all the major components of the simulated aerosol contain at least one functional group which has been implicated in mechanisms proposed for condensedphase association and accretion reactions. Thus, some degree of scaling would be expected to be necessary in almost every case, such that the assumption of a species-independent factor is believed to be a reasonable initial approximation.

The average empirical molecular composition of the simulated SOA for each case study event is indicated in Table 3. The empirical formulae and average molecular weights can be compared with those for Suwanee River fulvic acid, which was used by McFiggans et al. (2005) as an operational analogue for fine oxidised organic aerosol material in terms of its ability to reproduce the measured proton nuclear magnetic resonance $\left({ }^{1} \mathrm{H}\right.$ NMR) spectra of ambient aerosol samples. Suwanee River fulvic acid is a high molecular weight tetra-acid, possessing a combination of aromatic and aliphatic groups, with additional hydroxy and carbonyl groups, and with ether and ester linkages. Its molecular formula is $\mathrm{C}_{33} \mathrm{H}_{32} \mathrm{O}_{19}$ and its molecular weight is 732.6 $\mathrm{g} \mathrm{mol}^{-1}$. Recently, laser-desorption/ionisation mass spectrometric measurements have been made of organic aerosols in both chamber experiments and in the field. For example, Kalberer et al. (2004) recorded mass spectra which indicated that a substantial fraction of the organic aerosol produced from the photooxidation of 1,3,5-trimethylbenzene, in a reaction chamber, was composed of oligomeric material with molecular masses up to 1000 Dalton after $20 \mathrm{~h}$ reaction time. Similarly, Tolocka et al. (2004) recorded mass spectra (using matrix-assisted laser-desorption/ionisation spectrometry) of SOA samples produced from the ozonolysis of $\alpha$ pinene, again in a reaction chamber, and observed the substantial presence of oligomeric material with a wide range of molecular weights up to and above 700 Dalton. Finally, Samburova et al. (2005) observed mass spectrometric evidence of oligomeric, humic-like, substances, with upper limit molecular weights of 700 Dalton, from water-extracted aerosol samples collected in downtown Zurich, Switzerland. Given the reaction-types most likely to be responsible for the formation of high molecular weight oligomeric material (i.e., association reactions involving multifunctional carbonyl, and especially aldehyde, species - see, for example, Jang and Kamens, 2001a, 2001b; Jang et al., 2002; Gao et al., 2005; Liggio et al., 2005; Jang et al., 2005) the species represented in Fig. 1 constitute very suitable monomers: first, they physically partition to the condensed organic-phase to a significant degree, and, second, they variously possess the chemical functionalities required for carbonyl association reactions (e.g., hydroxy and hydroperoxy groups). It is also interesting to note that the various calculated ratios of organic mass to carbon mass (calculated as the average for all species comprising a given simulated SOA event - see Table 3) are similar to that for the operational SOA analogue species, Suwanee River fulvic acid (which has a mass ratio of 1.85), and also in agreement with the value of $(2.1 \pm 0.2)$ recommended for aged non-urban aerosols by Turpin and Lim (2001). It is expected that association reactions do not significantly alter the carbon to oxygen (and nitrogen) composition ratios in the products as compared with the sum of the reactants i.e. on forming a multifunctional adduct, from the association of two multifunctional reactants, the overall functionalgroup content is little changed after linkage. Accordingly, the simulated (atomic) ratios of carbon to oxygen (lying in the range 1.44-1.96) agree well with that of Suwanee River fulvic acid (1.74), suggesting that the simulated extents of oxidation are generally consistent with observation. The simulated aerosol composition also indicates contributions from oxidised nitrogen groups to SOA mass, with (atomic) ratios of carbon to nitrogen lying in the range 7.6-16.7. As indicated in Table 1, the simulated SOA includes important contributions from species containing nitro-, nitrate and peroxynitrate groups. The results therefore suggest that organic oxidised nitrogen potentially makes a notable contribution to the oxidised nitrogen content of ambient aerosols. 
Table 3. Empirical, average, molecular composition of simulated SOA.

\begin{tabular}{clcc}
\hline Trajectory $^{\mathrm{a}}$ & Average (empirical) formula of SOA $^{\mathrm{b}}$ & Average molecular weight of SOA $^{\mathrm{b}}$ & Organic mass/organic carbon mass $^{\mathrm{b}, \mathrm{c}}$ \\
\hline $\mathrm{A}$ & $\mathrm{C}_{6.23} \mathrm{H}_{7.37} \mathrm{O}_{3.98} \mathrm{~N}_{0.75}$ & 156.4 & 2.09 \\
$\mathrm{~B}$ & $\mathrm{C}_{6.30} \mathrm{H}_{7.83} \mathrm{O}_{3.83} \mathrm{~N}_{0.69}$ & 154.5 & 2.04 \\
$\mathrm{C}$ & $\mathrm{C}_{6.06} \mathrm{H}_{7.09} \mathrm{O}_{4.21} \mathrm{~N}_{0.80}$ & 158.5 & 2.18 \\
$\mathrm{D}$ & $\mathrm{C}_{6.46} \mathrm{H}_{8.54} \mathrm{O}_{3.97} \mathrm{~N}_{0.68}$ & 159.3 & 2.05 \\
$\mathrm{E}$ & $\mathrm{C}_{6.51} \mathrm{H}_{8.53} \mathrm{O}_{3.97} \mathrm{~N}_{0.70}$ & 160.1 & 2.05 \\
$\mathrm{~F}$ & $\mathrm{C}_{7.13} \mathrm{H}_{9.89} \mathrm{O}_{4.00} \mathrm{~N}_{0.55}$ & 167.4 & 1.96 \\
$\mathrm{G}$ & $\mathrm{C}_{7.28} \mathrm{H}_{10.22} \mathrm{O}_{4.00} \mathrm{~N}_{0.64}$ & 170.6 & 1.95 \\
$\mathrm{H}$ & $\mathrm{C}_{6.55} \mathrm{H}_{8.22} \mathrm{O}_{3.99} \mathrm{~N}_{0.72}$ & 161.0 & 2.04 \\
$\mathrm{I}$ & $\mathrm{C}_{6.68} \mathrm{H}_{8.48} \mathrm{O}_{4.12} \mathrm{~N}_{0.71}$ & 164.6 & 2.05 \\
$\mathrm{~J}$ & $\mathrm{C}_{7.57} \mathrm{H}_{11.53} \mathrm{O}_{3.86} \mathrm{~N}_{0.46}$ & 170.8 & 1.88 \\
$\mathrm{~K}$ & $\mathrm{C}_{7.53} \mathrm{H}_{11.37} \mathrm{O}_{3.98} \mathrm{~N}_{0.45}$ & 171.9 & 1.90 \\
\hline
\end{tabular}

a Letters correspond to trajectory arrival dates and times as described in the footnote of Table 1;

$\mathrm{b}$ calculated from the distribution of (monomeric; see text) oxidation products partitioned to the aerosol-phase;

$\mathrm{c}$ this is the ratio of the average molecular mass of the simulated SOA to the average mass of carbon in all species comprising the simulated SOA.

It can be seen in Tables 1 and 2, and Fig. 1, that between around 70 and 100 species represent $90 \%$ of the simulated SOA mass for all nine of the original case study events, and that these are derived, almost exclusively, from either aromatic or terpenic ( $\alpha$ - and $\beta$-pinene) precursors - see Fig. 2. Further to this, the great majority (90\%) of the aromatic contribution is due, in order of significance, to toluene, to the xylenes and to benzene. The relative importance of oxidation products from biogenic and anthropogenic precursors is also seen to be variable from one case study to the next of the original nine events, with, for example, the biogenic contribution ranging from 16.1 to $50.2 \%$. For the two additional case study events (trajectories $J$ and $K$; again, these events were selected due to simulations predicting that air masses travelling along these trajectories would be subjected to relatively-greater biogenic emissions) the biogenic source of SOA material is clearly dominant, providing around $85 \%$ of the total simulated mass of species partitioned to the condensed organic-phase. The simulated SOA for these latter two events contain a number of other multifunctional species derived from $\alpha$ - and $\beta$-pinene which are not included in Fig. 1, most notably C811NO3 (2,2-dimethyl-3nitrooxymethyl-cyclobutanecarboxylic acid) and APINBCO (2-hydroxy-2,6,6-trimethyl-bicyclo[3.1.1]heptan-3-one).

\subsection{Sensitivity of simulated SOA mass to changes in VOC emissions}

Having investigated the detailed chemical composition of simulated SOA material for various case study events, and having determined which emitted VOC species (or category of VOC) the predominant particle-phase species are derived from, the sensitivity of the simulated SOA mass to changes in the emission rates of primary VOC pollutants were assessed.
This was carried out by re-running each of the 11 air trajectories indicated in Table 1: first, with the total anthropogenic VOC emission rate increased by $10 \%$ (compared to the original set of simulations), and, second, with the total biogenic VOC emission rate increased by $10 \%$. The results of these simulations are summarised in Table 4. The proportional, per cent, increases in the mass of SOA (relative to the masses listed in Table 1 - i.e. the "base case" simulations) have been calculated for each trajectory with, in turn, increased anthropogenic and biogenic emissions ( $\alpha$ and $\beta$ respectively). By further expressing the per cent increase in SOA mass due to increased anthropogenic emissions (again, $\alpha$ ) as a proportion of the total (summed) per cent increase in SOA mass due to changes in the emission rates of all VOC (i.e. $\alpha /(\alpha+\beta))$ it may be expected that a relative measure of the anthropogenic content of the SOA is obtained. It is noted that this expectation assumes that the simulated increase in SOA mass in each sensitivity study results predominantly from the incremental increases in the concentrations of products derived from the oxidation of either anthropogenic or biogenic VOC. Although it is recognised that the increased SOA mass in each case promotes additional transfer of partitioning species derived from both anthropogenic and biogenic precursors (i.e., the system is not strictly linear), additional sensitivity tests in which the emissions of both anthropogenic VOC and biogenic VOC were increased by $10 \%$ simultaneously resulted in SOA increments which were only slightly greater (by an average factor of ca. 1.06) than the sum of the SOA increments when anthropogenic VOC and biogenic VOC emissions were increased independently. As a result it is concluded that the main contributor to the increase in SOA formation in each of the independent cases is the incrementally increased concentration of VOC oxidation products resulting from either anthropogenic or biogenic precursors, such that 

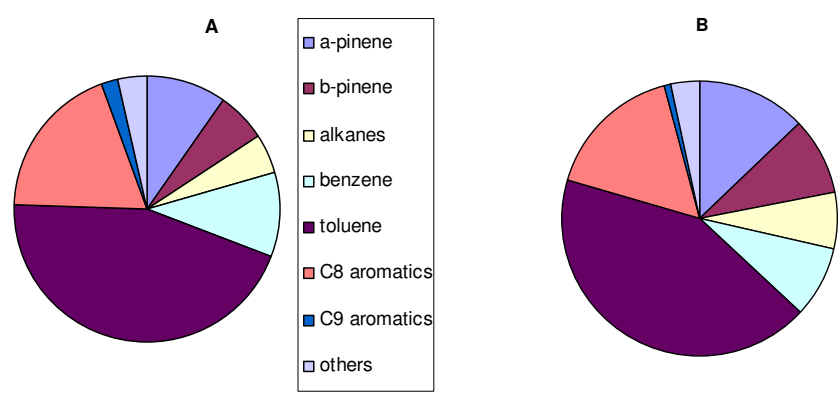

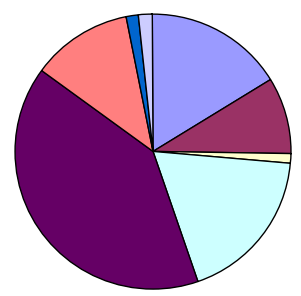

$\mathbf{F}$
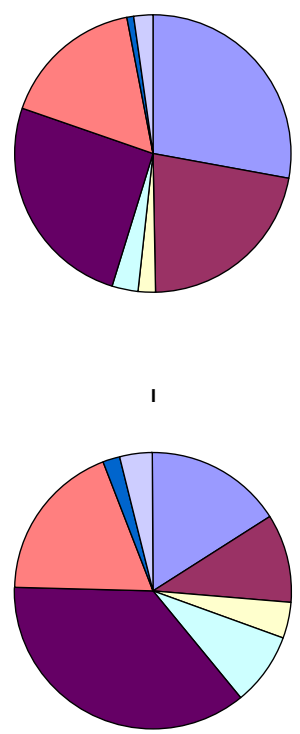

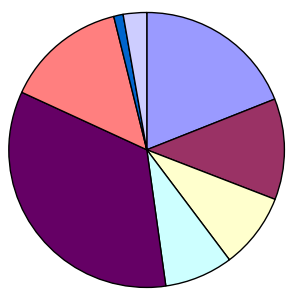

G

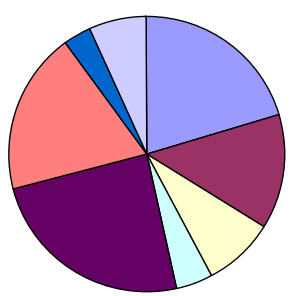

J

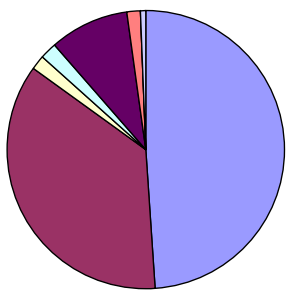

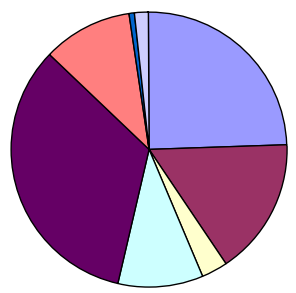

H

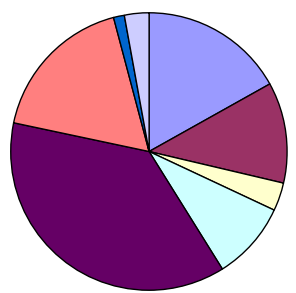

K

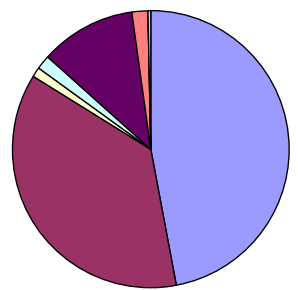

Fig. 2. Distribution of species contributing to the simulated mass of SOA (categorised by parent VOC). Note that $\alpha$ - and $\beta$-pinene are used to represent all emitted monoterpenes (see Johnson et al., 2006).

the increased gas-to-aerosol transfer of material in general at the slightly higher OA concentrations is a secondary effect. As a result, the quantity $\alpha /(\alpha+\beta)$ provides a measure of the anthropogenic contribution to SOA formation, to a good first approximation.

This "metric" of anthropogenic content is plotted in Fig. 3 against the anthropogenic contributions calculated, for the base case simulations, from the compositional data in Ta- ble 1 and as listed in Table 2. As can be seen in this figure, the sensitivity of simulated SOA mass to changes in the emission rates of anthropogenic, and biogenic, VOC appears to be consistent with the simulated detailed aerosol composition, for all events. In the case studies considered, the different SOA contributions of anthropogenic and biogenic precursors are determined by the histories of the various air masses, as represented by different air trajectories. For example, air 
Table 4. Results of tests of the sensitivity of simulated SOA mass, for each trajectory studied, to changes in the emission rates of primary emitted VOC (anthropogenic and biogenic).

\begin{tabular}{lccccccccccc}
\hline & \multicolumn{10}{c}{ Trajectory $^{\mathrm{a}}$} \\
\hline & $\mathrm{A}$ & $\mathrm{B}$ & $\mathrm{C}$ & $\mathrm{D}$ & $\mathrm{E}$ & $\mathrm{F}$ & $\mathrm{G}$ & $\mathrm{H}$ & $\mathrm{I}$ & $\mathrm{J}$ & $\mathrm{K}$ \\
\hline $\mathrm{SOA}\left(1.0 \times \mathrm{E}_{\mathrm{VOC}}\right) / \mu \mathrm{g} \mathrm{m}^{-3 \mathrm{~b}}$ & 0.507 & 1.63 & 1.08 & 5.01 & 1.11 & 0.216 & 0.788 & 0.294 & 0.234 & 19.3 & 4.61 \\
$\mathrm{SOA}\left(1.1 \times \mathrm{E}_{\mathrm{AVOC}}\right) / \mu \mathrm{g} \mathrm{m}^{-3 \mathrm{c}}$ & 0.542 & 1.75 & 1.15 & 5.55 & 1.15 & 0.220 & 0.844 & 0.309 & 0.246 & 20.0 & 4.72 \\
$\mathrm{SOA}\left(1.1 \times \mathrm{E}_{\mathrm{BVOC}}\right) / \mu \mathrm{g} \mathrm{m}^{-3 \mathrm{~d}}$ & 0.515 & 1.66 & 1.11 & 5.22 & 1.15 & 0.222 & 0.815 & 0.301 & 0.240 & 22.3 & 5.46 \\
$\left(\alpha=\Delta \mathrm{SOA}_{\mathrm{AVOC}}\right) / \% \mathrm{e}$ & 6.91 & 7.36 & 6.48 & 10.8 & 3.60 & 1.85 & 7.11 & 5.10 & 5.13 & 3.62 & 2.39 \\
$\left(\beta=\Delta \mathrm{SOA}_{\mathrm{BVOC}}\right) / \% \mathrm{f}$ & 1.58 & 1.84 & 2.78 & 4.19 & 3.60 & 2.78 & 3.43 & 2.38 & 2.56 & 15.5 & 18.4 \\
$((\alpha /(\alpha+\beta)) \times 100) / \% \mathrm{~g}$ & 81.4 & 80.0 & 70.0 & 72.0 & 50.0 & 40.0 & 67.5 & 68.2 & 66.7 & 18.9 & 11.5 \\
$\Sigma($ Anthropogenic $) / \% \mathrm{~h}^{\mathrm{h}}$ & 83.9 & 77.8 & 74.7 & 69.2 & 59.5 & 49.8 & 66.2 & 71.3 & 73.5 & 15.0 & 16.0 \\
\hline
\end{tabular}

a Letters correspond to trajectory arrival dates and times as described in the footnote of Table 1;

$\mathrm{b}$ simulated mass concentration of SOA mixing ratio for emissions base case (see text): data as listed in Table 1;

$\mathrm{c}$ mass concentration of SOA mixing ratio for simulation with $10 \%$ increase in emission rates of all primary anthropogenic VOC;

$\mathrm{d}$ mass concentration of SOA mixing ratio for simulation with $10 \%$ increase in emission rates of all primary biogenic VOC;

e $\triangle \mathrm{SOA}_{\mathrm{AVOC}}=\left[\left(\mathrm{SOA}\left(1.1 \times \mathrm{E}_{\mathrm{AVOC}}\right) \div \mathrm{SOA}\left(1.0 \times \mathrm{E}_{\mathrm{VOC}}\right)\right) \times 100\right]-100$

f $\triangle \mathrm{SOA}_{\mathrm{BVOC}}=\left[\left(\mathrm{SOA}\left(1.1 \times \mathrm{E}_{\mathrm{BVOC}}\right) \div \mathrm{SOA}\left(1.0 \times \mathrm{E}_{\mathrm{VOC}}\right)\right) \times 100\right]-100$

$\mathrm{g}$ this corresponds to the proportion of the total (summed) sensitivity in simulated SOA mass that is attributable to changes in the emission rates of anthropogenic VOC;

$\mathrm{h}$ per cent contribution of species derived from anthropogenic VOC to the total mass of SOA for the base case simulations (data taken from bottom row of Table 2).

arriving at the measurement site at 18:00 on 6 August 2003 (trajectory D) is simulated to have originated in the North Sea before passing in a clockwise sweep through Denmark, Germany, the Netherlands, Belgium, France and the UK, eventually arriving at the measurement site from the West. During this journey, the air has passed over areas with significant human populations, significant industry and some forest coverage - thus the air has been subjected to significant emissions of anthropogenic VOC and $\mathrm{NO}_{\mathrm{x}}$, and some input of biogenic VOC. The significant mass concentration of SOA simulated for this event (ca. $5 \mu \mathrm{g} \mathrm{m}^{-3}$ ) is predominantly composed of species derived from the photooxidation of anthropogenic VOC. By contrast, air arriving at 12:00 on 7 August 2003 (trajectory $J$ ) is simulated to originate in North Eastern France and to sweep, clockwise, across Northern France, South Western England and Wales, before passing over the Midlands of England and approaching the measurement site from the North West. Again, the air mass passes over areas with significant anthropogenic emissions of VOC and $\mathrm{NO}_{\mathrm{x}}$ in addition to relatively sparsely-populated areas with more significant forest coverage (e.g. North Eastern France, South Western England and Wales). Thus, the air is subjected to significant emissions of VOC with a larger proportion coming from biogenic sources. As for 6 August 2003 (trajectory D), a significant mass concentration of SOA is simulated (ca. $20 \mu \mathrm{g} \mathrm{m}^{-3}$ ) but with a dominant content of species derived from the atmospheric oxidation of biogenic VOC.

A major conclusion of the companion paper was that, owing to SOA formation resulting from the atmospheric oxi-

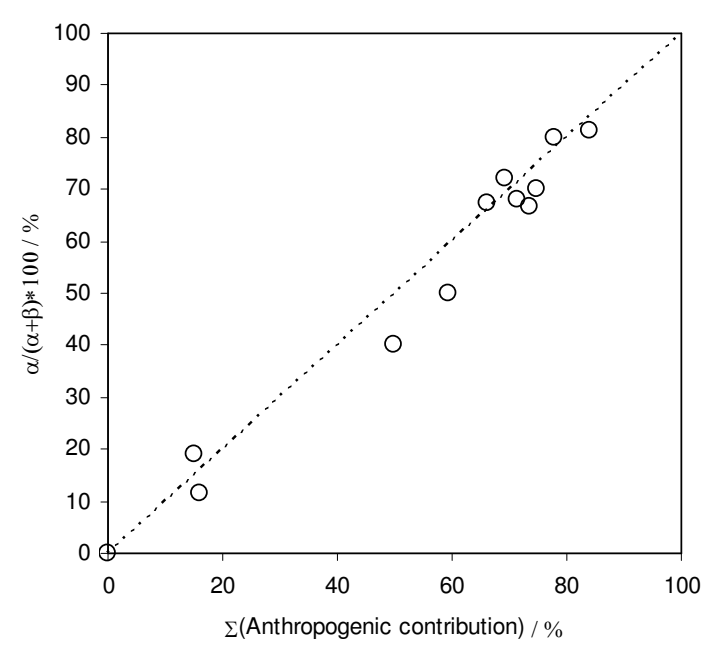

Fig. 3. Plot of the ratio of (proportional) increase in SOA mass due to increased anthropogenic emissions to the summed increase in SOA due to increased anthropogenic and biogenic emissions $(=\alpha /(\alpha+\beta)$, see text $)$ versus the per cent anthropogenic contribution of species to SOA for each base case simulation.

dation of VOC, the growth of OA material is a regionalscale phenomenon. Evidence for this was provided in the form of a strong correlation between organic aerosol mass concentrations and ozone volume mixing ratios; both measured at the Writtle college measurements site, for the 2003 TORCH campaign (see Fig. 7 of Johnson et al., 2006). As stated in the companion paper, it is well established that 
elevated ozone concentrations in the UK are generated by regional-scale VOC oxidation under anticyclonic conditions in north-west Europe (e.g., Derwent et al., 1996; Jenkin et al., 2002). Thus, where increased emissions of VOC resulted in increased simulated concentrations of OA, at the end of $96 \mathrm{~h}$ air trajectories, a similar effect is expected, and indeed was observed, for simulated ozone concentrations. Estimates of the anthropogenic contribution to photochemical ozone formation, made in an analogous manner to that for SOA formation, were found to be similar to the estimated SOA contributions, for each trajectory, except that the former contributions were generally larger than the latter. This is not surprising given the fact that a larger subset of all emitted anthropogenic VOC will contribute to photochemical ozone formation - this being a function of individual VOC emission rates and reactivities. By contrast, a relatively-smaller subset of all emitted VOC will contribute to SOA formation simply as a result of the requirement that sufficiently nonvolatile oxidation products are formed in order to transfer to the condensed organic-phase. This is clearly seen in the relatively small number of emitted VOC which are identified as SOA precursors in the present study.

\section{Conclusions}

A photochemical trajectory model has been operated with a highly-detailed gas-phase mechanism for the atmospheric degradation of VOC pollutants (the MCM v3.1) incorporating a representation of the transfer of semi- and nonvolatile oxidation products to a condensed particle-phase. As described in the companion to this paper (Johnson et al., 2006), the combined model has been used to simulate the formation of SOA during the first TORCH field measurement campaign, in the Southern UK in August 2003. In the present investigation, the detailed chemical composition of the simulated SOA has been determined and the constituent species have been attributed to the primary (emitted) VOC from which they are derived. For different case study events the mass contributions of products derived from anthropogenic and biogenic VOC are relatively different. The anthropogenic contribution varies from ca. $85 \%$ to ca. $15 \%$, depending on the geographical history of the air mass arriving at the measurement site and hence the pollutant emissions it experienced during the simulated $96 \mathrm{~h}$ period. The sensitivity of the simulated mass of SOA, for the 11 air trajectories studied, has been investigated in terms of increased $(+10 \%)$ emissions of anthropogenic and biogenic VOC. The relative sensitivities, to increased anthropogenic and biogenic emissions, are consistent with the speciation of the simulated SOA material and the primary VOC from which these are derived.

Acknowledgements. The work described in this paper was funded by the Natural Environment Research Council, (NERC), as part of the Tropospheric Organic Chemistry Experiment (TORCH) under contract NER/T/S2002/00495, and by the Department for Environment, Food and Rural Affairs (DEFRA) under contract EPG 1/3/200. MEJ also acknowledges NERC for support via a Senior Research Fellowship (NER/K/S/2000/00870).

Edited by: F. J. Dentener

\section{References}

Alfarra, M. R.: Insights into atmospheric organic aerosols using an aerosol mass spectrometer, Ph.D. Thesis, University of Manchester, 2004.

Allan, J. D., Alfarra, M. R., Bower, K. N., Williams, P. I., Gallagher, M. W., Jimenez, J. L., McDonald, A. G., Nemitz, E., Canagaratna, M. R., Jayne, J. T., Coe, H., and Worsnop, D. R.: Quantitative Sampling Using an Aerodyne Aerosol Mass Spectrometer - 2. Measurements of Fine Particulate Chemical Composition in Two U.K. Cities, J. Geophys. Res., 108(D3), 4091, doi:10.1029/2002JD002359, 2003.

Anderson, H. R., Bremner, S. A., Atkinson, R. W., Harrison, R. M., and Walters, S.: Particulate matter and daily mortality and hospital admissions in the west midlands conurbation of the United Kingdom: associations with fine and coarse particles, black smoke and sulphate, Occupational and Environmental Medicine, 58, 504-510, 2001.

AQEG: Nitrogen dioxide in the United Kingdom. Report of the UK Air Quality Expert Group, prepared at the request of the Department for Environment Food and Rural Affairs, London, PB9025, 2004.

AQEG: Particulate matter in the United Kingdom, Report of the UK Air Quality Expert Group. Prepared at the request of the Department for Environment Food and Rural Affairs, London, PB10580 ISBN 0-85521-143-1, 2005.

Barsanti K. C. and Pankow J. F.: Thermodynamics of the formation of atmospheric particulate matter by accretion reactions - Part 1 : Aldehydes and ketones, Atmos. Environ., 38, 4371-4382, 1996.

Bloss, C., Wagner, V., Jenkin, M. E., Volkamer, R., Bloss, W. J., Lee, J. D., Heard, D. E., Wirtz, K., Martin-Reviejo, M., Rea, G., Wenger, J. C., and Pilling, M. J.: Development of a detailed chemical mechanism (MCMv3.1) for the atmospheric oxidation of aromatic hydrocarbons, Atmos. Chem. Phys., 5, 641644, 2005,

SRef-ID: 1680-7324/acp/2005-5-641.

Derwent, R. G., Jenkin, M. E., and Saunders, S. M.: Photochemical ozone creation potentials for a large number of reactive hydrocarbons under European conditions, Atmos. Environ., 30, 181-199, 1996.

Dore, C., Hayman, G., Scholefield, P., Hewitt, N., Winiwarter, W., and Kressler, F.: Mapping of biogenic VOC emissions in England and Wales. Environment Agency R\&D Technical Report E1-122/TR, ISBN 1-84432-092-8, 2003.

Englert, N.: Fine particles and human health - a review of epidemiological studies, Toxicology Letters, 149, 235-242, 2004.

Finlayson-Pitts, B. J. and Pitts, J. N.: Chemistry of the upper and lower atmosphere: Theory, experiments and applications, Academic Press, San Diego, 1999.

Gao, S., Ng, N. L., Keywood, M., Varutbangkul, V., Bahreini, R., Nenes, A., He, J., Yoo, K. Y., Beauchamp, J. L., Hodyss, R. P., Flagan, R. C., and Seinfeld, J. H.: Particle phase acidity and 
oligomer formation in secondary organic aerosol, Environ. Sci. Technol., 39, 6582-6589, 2005.

Intergovernmental Panel on Climate Change (IPCC): Climate change: The scientific basis, Cambridge University Press, UK, 2001

Jang, M. S. and Kamens, R. M.: Characterization of secondary aerosol from the photooxidation of toluene in the presence of $\mathrm{NO}_{\mathrm{x}}$ and 1-propene, Environ. Sci. Technol., 35, 3626-3639, 2001a.

Jang, M. and Kamens, R. M.: Atmospheric secondary organic aerosol formation by heterogeneous reactions of aldehydes in the presence of sulphuric acid aerosol catalyst, Environ. Sci. Technol., 35, 4758-4766, 2001b.

Jang, M., Czoschke, N. M., Lee, S., and Kamens, R. M.: Heterogeneous atmospheric aerosol production by acid-catalyzed particlephase reactions, Science, 298, 814-817, 2002.

Jang, M., Czoschke, N. M., and Northcross, A. L.: Semiempirical model for organic aerosol growth by acid-catalyzed heterogeneous reactions of organic carbonyls, Environ. Sci. Technol., 39, 164-174, 2005.

Jenkin, M. E., Saunders, S. M., and Pilling, M. J.: The tropospheric degradation of volatile organic compounds: a protocol for mechanism development, Atmos. Environ., 31, 81-104, 1997.

Jenkin, M. E., Davies, T. J., and Stedman, J. R.: The origin and day-of-week dependence of photochemical episodes in the UK, Atmos. Environ., 36, 999-1012, 2002.

Jenkin, M. E., Saunders, S. M., Wagner, V., and Pilling, M. J.: Protocol for the development of the Master Chemical Mechanism, MCM v3 (Part B): tropospheric degradation of aromatic volatile organic compounds, Atmos. Chem. Phys., 3, 181-193, 2003,

SRef-ID: 1680-7324/acp/2003-3-181.

Jenkin, M. E.: Modelling the formation and composition of secondary organic aerosol from $\alpha$ - and $\beta$-pinene ozonolysis using MCM v3, Atmos. Chem. Phys., 4, 1741-1757, 2004,

\section{SRef-ID: 1680-7324/acp/2004-4-1741}

Joback, K. G. and Reid, R. C: Estimation of pure-component properties from group-contributions, Chem. Eng. Commun., 57, 233243, 1987

Johnson, D., Jenkin, M. E., Wirtz, K., and Martin-Reviejo, M.: Simulating the formation of secondary organic aerosol from the photooxidation of toluene, Environ. Chem., 1, 150-165, 2004.

Johnson, D., Jenkin, M. E., Wirtz, K., and Martin-Reviejo, M.: Simulating the formation of secondary organic aerosol from the photooxidation of aromatic hydrocarbons, Environ. Chem., 2, 3548, 2005.

Johnson, D., Utembe, S. R., Jenkin, M. E., Derwent, R. G., Hayman, G. D, Alfarra, M. R., Coe, H., and McFiggans, G.: Simulating regional scale secondary organic aerosol formation during the TORCH 2003 campaign in the southern UK, Atmos. Chem. Phys., 6, 403-418, 2006, SRef-ID: 1680-7324/acp/2006-6-403.

Kalberer, M., Paulsen, D., Sax, M., Steinbacher, M., Dommen, J., Prevot, A. S. H., Fisshea, R., Weingartner, E., Frankenvich, V., Zenobi, R., and Baltensperger, U.: Identification of polymers as major components of atmospheric organic aerosols, Science, 303, 1659-1662, 2004.

Kamens, R. M., Jang, M., Chien, C.-J., and Leach, K.: Aerosol formation from the reaction of $\alpha$ tpinene and ozone using a gasphase kinetics-aerosol partitioning model, Environ. Sci. Technol., 33, 1430-1438, 1999.
Kanakidou, M., Seinfeld, J. H., Pandis, S. N., Barnes, I., Dentener, F. J., Facchini, M. C., Van Dingenen, R., Ervens, B., Nenes, A., Nielsen, C. J., Swietlicki, E., Putaud, J. P., Balkanski, Y., Fuzzi, S., Hjorth, J., Moortgat, G. K., Winterhalter, R., Myhre, C. E. L., Tsigaridis, K., Vignati, E., Stephanou, E. G., and Wilson, J.: Organic aerosol and global climate modelling: a review, Atmos. Chem. Phys., 5, 1053-1123, 2005,

SRef-ID: 1680-7324/acp/2005-5-1053.

Laden, F., Neas, L. M., Dockery, D. W., and Schwartz J.: Association of fine particulate matter from different sources with daily mortality in six US cities, Environ. Health Perspect., 108, 941947, 2000.

Liggio, J., Li, S.-M., and McLaren, R.: Heterogeneous reactions of glyoxal on particulate matter: Identification of acetals and sulfate esters, Environ. Sci. Technol., 39, 1532-1541, 2005.

McFiggans, G., Alfarra, M. R., Allan, J., Bower, K., Coe, H., Cubison, M., Topping, D., Williams, P., Descari, S., Facchini, C., and Fuzzi, S.: Simplification of the representation of the organic component of atmospheric particulates, Faraday Discuss., 130, 341-362, 2005.

Pankow, J. F.: An absorption model of gas/particle partitioning involved in the formation of secondary organic aerosol, Atmos. Environ., 28, 189-193, 1994.

Samburova, V., Kalberer, M., and Zenobi, R.: Characterization of high molecular weight compounds in urban atmospheric particles, Atmos. Chem. Phys., 5, 2163-2170, 2005,

SRef-ID: 1680-7324/acp/2005-5-2163.

Saunders, S. M., Jenkin, M. E., Derwent, R. G., and Pilling, M J.: Protocol for the development of the Master Chemical Mechanism, MCM v3 (Part A): tropospheric degradation of nonaromatic volatile organic compounds, Atmos. Chem. Phys., 3, 161-180, 2003,

SRef-ID: 1680-7324/acp/2003-3-161.

Schwartz, J., Dockery, D. W., and Neas, L. M.: Is daily mortality associated specifically with fine particles?, J. Air Waste Manage. Assoc., 46, 927-939, 1996.

Seinfeld, J. H. and Pandis, S. N.: Atmospheric chemistry and physics: from air pollution to climate change, WileyInterscience, New York, 1998.

Seinfeld, J. H. and Pankow, J. F.: Organic atmospheric particulate material, Ann. Rev. Phys. Chem., 54, 121-140, 2003.

Stein, S. E. and Brown, R. L.: Estimation of normal boiling points from group contributions, J. Chem. Info. Comp. Sci., 34, 581587, 1994.

Tobias, H. J. and Ziemann, P. J.: Thermal desorption mass spectrometric analysis of organic aerosol formed from reactions of 1-tetradecene and $\mathrm{O}_{3}$ in the presence of alcohols and carboxylic acids, Environ. Sci. Technol., 34, 2105-2115, 2000.

Tolocka, M. P., Jang, M., Ginter, J. M., Cox, F. J., Kamens, R. M., and Johnston, M. V.: Formation of oligomers in secondary organic aerosol, Environ. Sci. Technol., 38, 1428-1434, 2004.

Turpin, B. J. and Lim, H.-J.: Species contributions to PM2.5 mass concentrations: revisiting common assumptions for estimating organic mass, Aerosol. Sci. Technol., 35, 602-610, 2001.

Utembe, S. R., Jenkin, M. E., Derwent, R. G., Lewis, A. C., Hopkins, J. R., and Hamilton, J. F.: Modelling the ambient distribution of organic compounds during the August 2003 ozone episode in the southern UK, Faraday Discuss., 130, 311-326, 2005. 